\title{
Concordance of whole-slide digital imaging and conventional microscopy for routine assessment of gastrointestinal surgical pathology specimens
}

\begin{abstract}
Abbreviations: GI, gastrointestinal; UFS, ultra-fast scanner; IMS, image management system

Histopathology is a rapidly advancing science and requires a great deal of sophistication in terms of equipment and special stains with the need for subspecialisation. The current workload in the NHS does not allow speciality reporting throughout the entire country. There are, however experts in the field in all specialities who can report the difficult cases. Digital pathology has seen, in recent years, a revolution in its use ranging from routine reporting to remote consultation and in quality assurance programmes.
\end{abstract}

The aim is to assess the concordance of surgical pathology specimens reporting between conventional microscopy and digital technology using the whole-slide imaging scanning technique.

A pilot study using the Philips Digital Pathology Solution to study cases of gastrointestinal (GI) pathology and compared with conventional microscopic assessment. Twenty-nine GI cases were chosen from the files by one of the authors (CG) who selected some old cases reported by two GI pathologists (NH and ES). Both pathologists reported again their own cases anonymised and coded by CG. The cases were a mixture of gallbladder, oesophageal, duodenal, gastric and colonic biopsies. The results were matched with the original report as reported under the microscope. All the slides used for this study were scanned using the Philips Ultra-Fast Scanner (UFS), which is a digital slide scanner capable of scanning glass slides at $40 \mathrm{x}$ magnification. Each slide takes approximately 60 seconds to scan and is then available for pathologists to view. The authors of this paper were given log-in details to view the images using a web-link to the Philips Image Management System (IMS). These images can then be accessed anytime, anywhere facilitating expert opinion and ultimately resulting in faster reporting time than using traditional methodology.

Twenty-nine cases (14 by ES and 15 by $\mathrm{NH}$ ) were reviewed using the Philips Digital Imaging software. The cases included 11 large bowel biopsies, 3 duodenal biopsies, 5 esophageal biopsies, 6 gallbladders and 4 gastric biopsies reported between September to December 2013.

In 25 cases, there were complete agreement between the original $\mathrm{H}$ and $\mathrm{E}$ slides and the digital imaging diagnosis. These cases included Barrett's esophagus, normal large bowel biopsies, gallbladders with inflammation and gastric biopsies with inflammation and one gastric tubular adenoma.

Six cases showed disagreement between the H\&E slides and the digital imaging diagnosis (originally reported by one pathologist). These included 2 large bowel biopsies with melanosis coli and muciphages within the lamina propria, 2 duodenal biopsies and 2 large bowel biopsies with intraepithelial lymphocytosis that were called normal on digital imaging analysis.

There was complete agreement in $80 \%$ of cases; however, in $20 \%$ of cases there was disagreement between the H\&E diagnosis and
Volume I Issue 2 - 2014

\author{
Emil Salmo, ${ }^{1,2}$ Najib Haboubi, ${ }^{2,3}$ Claire \\ Grinnell, ${ }^{3}$ Andy Humes ${ }^{4}$ \\ 'Histopathology Department, Royal Bolton Hospital, United \\ Kingdom \\ ${ }^{2}$ Histopathology Department, Spire Manchester Hospital, United \\ Kingdom \\ ${ }^{3}$ Histopathology, University of South Manchester, United \\ Kingdom \\ ${ }^{4}$ Digital Imaging, Philips, Netherlands
}

Correspondence: Emil Salmo, Histopathology Department, Royal Bolton Hospital, Minerva Road, Bolton, BL4 0JR, United Kingdom, Tel 00-44-740300 I I I I,Email emilsalmo@hotmail.com

Received: July 08, 2014 | Published: July 28, 2014

the digital imaging analysis of the cases all of which included subtle changes such as melanosis coli and intraepithelial lymphocytosis.

Digital pathology and whole slide imaging techniques have seen increasing use in recent years for a variety of purposes ranging from routing reporting to remote pathology consultation. ${ }^{1}$ In a review by Ghaznavi et al., ${ }^{1}$ they showed that it is particularly useful in quality assurance programmes and as a teaching material where conferences are held in remote areas. Pantanowitz et al. $^{2}$ in their study also supported this view.

In our small pilot study, most of the cases were reported with ease and showed complete concordance with the original H\&E diagnosis. However, one of the problems encountered is the speed of the internet connection needed for a good pixilation of pictures, which was suboptimal in one of the sites.

In our small study, we feel that for the majority of pathology cases there was no difference between the microscopic and digital assessment. However, most of the cases that showed disconcordance between the two methods are subtle changes, included melanosis, and intraepithelial lymphocytosis. Although, melanosis coli, in most cases, does not bear a management consequence to the patient, the inability to spot intraepithelial lymphocytes in the digital images showed that these subtle changes can easily be missed on the screen. This was due to poor image quality especially on high power examination which can be attributed to the speed of internet connection, although all these changes were detected in the original $\mathrm{H}$ and $\mathrm{E}$ slides. Hence, for these features, the pathologists may need a learning curve to spot out these subtleties as missing these changes can alter the management of patients and miss early changes of Celiac disease. A larger study is required to further evaluate the Philips Digital Pathology Solution. 


\section{Conflicts of Interest}

The author declares that there is no conflicts of interest.

\section{Acknowledgements}

None.

\section{Funding}

None.

\section{References}

1. Ghaznavi F, Evans A, Madabhushi A, et al. Digital imaging in pathology: whole-slide imaging and beyond. Annu Rev Pathol. 2013;8: 331-359.

2. Pantanowitz L, Valenstein PN, Evans AJ, et al. Review of the current state of whole slide imaging in pathology. J Pathol Inform. 2010;2:36. 